\title{
Opportunities of Digital Technologies as a Tool for Improving and Personalizing Specialized Nutrition
}

\author{
Aleksandra Utkina*, and Vasiliy Karagodin \\ Plekhanov Russian University of Economics, 117997 Moscow, Russia
}

\begin{abstract}
The possibilities of nutrigenomics as an approach to determining the effectiveness of specialized food products have been studied. For this purpose, the influence of consumption of whey protein concentrate FitPROTEIN and the carrier of branched-chain amino acids SPORTAMIN ${ }^{\circ} B C A A 6000$ on the expression of the target gene FTO was determined. Despite a certain similarity in the composition of these products, their effect on the average level of FTO gene expression is significantly different. The results obtained are discussed from the standpoint of identification of the target gene, detection of gene polymorphism, analysis of expression data and the possibility of updating these procedures using digital technologies. An algorithm for evaluating the effectiveness of specialized food products based on nutrigenomic studies is proposed. Nevertheless, it seems promising to transfer experimentation to the virtual sphere through the use of digital twins, including in combination with the Internet of Things.
\end{abstract}

\section{Introduction}

As is known, the world market for health-improving, functional and sports food products is growing steadily, especially in Western countries. For Russia, it also has an extremely important social and economic significance, including in connection with insufficient consumption of vital macro- and micronutrients.

However, nowadays, it is rather possible to state the stagnation of the domestic market for that part of specialized food products (SFPs) that could be used for health-improving and prophylactic purposes and potentiate a person's physical capabilities.

There are many reasons for this state of affairs (terminological confusion, imperfect legal and regulatory documentation, poor information and educational support, etc.), but the main problem is, obviously, the lack of reliably confirmed efficiency of the SFPs. For instance, more than 20,000 nutritional supplements and about a thousand sports nutrition products are registered in the Russian Federation, and the effectiveness of evidence-based medicine was studied only in isolated cases for these products. Most likely, due to the duration and high cost of clinical trials, this situation will continue in the foreseeable future.

\footnotetext{
*Corresponding author: ytkinaas@yandex.ru
} 
At the same time, millions of rubles of state and commercial funds were spent on the development of SFPs, and the life cycle of most of these products ended soon after their registration. Moreover, social and moral damage is also important when, under the influence of advertising, a consumer acquires an empty shell.

In their composition, SFPs are analogs of multicomponent drugs, having an active principle represented by several ingredients. Thus, for each of them, it is necessary to take into account the dose-effect relationship, and the duration of exposure to the human body, and bioavailability, and other aspects faced by clinical pharmacology. Furthermore, genomicproteomic analysis made it possible not only to decipher the human genome, but also to begin studies of the nutrient-dependent regulation of gene expression, including in individuals from risk groups of non-infectious chronic diseases and special conditions of activity, in particular, sports.

It seems to us that the current state of biomedical science in combination with digital technologies allows us to propose new approaches to confirming the effectiveness of SFPs, including at the level of individual consumption. One of these approaches has received experimental confirmation, the results of which are presented in this article.

\section{Materials and methods}

Two SFPs used in the experiments as sources of amino acids, Whey FitPROTEIN (whey protein concentrate) and SPORTAMIN® BCAA 6000 (BCAA carrier) were purchased from the Russian company ACADEMIA-T and consumed once daily for three days in the dose recommended by the manufacturer $\left(20 \mathrm{~g}\right.$ for Whey FitPROTEIN and $6 \mathrm{~g}$ for SPORTAMIN ${ }^{\circledR}$ BCAA 6000). Scrapings of epithelial cells of the oral mucosa, obtained as described in [1], served as biological material. The study involved 30 male volunteers aged 20-25 years, leading a healthy lifestyle and not suffering from non-infectious chronic diseases. They were divided into 2 groups of 15 people, and the members of each group took one of the SFPs in the same dose for 3 days. The biological material from each of the subjects was taken for research before the start of taking the product (control), and then six times at the intervals indicated below.

The target gene for SFP (FTO) was selected based on the analysis of literature data on nutrigenetics, taking into account the role of these SFPs in metabolism and participation in providing the main expected effects.

The effect of SFPs on FTO gene expression was studied using the generally accepted procedure [2], namely, by combining DNA microanalysis and real-time polymerase chain reaction (PCR). DNA and RNA were isolated from biological material using reagent kits for the extraction of nucleic acids (Syntol, Russia). Total RNA was isolated from biological samples using the PureLink RNA Mini Kit (Thermo Fisher Scientific, USA) according to the manufacturer's instructions. The reverse transcription reaction to obtain complementary DNA (cDNA) on the RNA template was performed using the iScript RT Supermix kit (BioRad, USA). The resulting total RNA of each sample was converted into complementary DNA (cDNA) for further analysis on an amplifier using a set of reagents for reverse transcription and a thermostat. The analysis of the expression of the studied genes was performed by reverse transcription PCR with gene-specific primers TaqMan Gene Expression Assays (Applied Biosystems) with primers TaqMan ${ }^{\mathrm{TM}}$ Gene Expression Assays (Applied Biosystems). cDNA synthesis was performed using the Maxima First Strand cDNA Synthesis Kit (Thermo Scientific). The expression level of the studied gene was normalized relative to the expression of the "housekeeping" gene GAPDH (Applied Biosystems). As an internal control, we analyzed the expression of the reference "housekeeping" gene, which was the TATA-box binding protein gene. The detection of fluorescence, as well as the primary processing of the results, was carried out using the CFX Manager 3.1 software for 
CFX96 apparatus by BIO-RAD (USA) in an automatic mode. Statistical processing of the research results was carried out using the SPSS Statistics 27.0 software.

\section{Results and discussion}

An important feature of SFPs, which distinguishes them from traditional food, but brings them closer to drugs, is their effectiveness. As is already known, personalized medicine is trying to solve the problem of the variability of the effect of drugs on the human body, including with the help of digital technologies. It is logical to expect that the effects of the same SFPs for different people may differ significantly.

The choice of the most effective health-improving, prophylactic or therapeutic agent from many similar ones could be made using such a tool as a person's digital twin [3]. It makes it possible to virtually immerse a drug molecule in a digital copy of a person, simulate the distribution of a drug throughout the body, and observe other physiological effects without performing field tests.

Probably, a similar scheme could be used to assess the SFPs, but, unfortunately, the creation of a person's digital twin has not yet taken place, although a digital twin of the heart already exists (Dassault). The computer program developed by the authors of this project makes it possible to turn a 2D scan of a real human heart into a multidimensional digital twin that simulates all the features of the organ functioning. Modeling the functioning of a gene seems to be even more promising, and in this regard, one should point out the study of gene expression in the laboratory of Professor Wong from California [4], who is consistently moving towards this goal.

The development of molecular genetic approaches to the study of the mechanisms of action of nutrients on biological objects has become the basis for conducting our own experiments in which the ability of the SFPs to influence the expression of target genes was determined. In particular, two popular Russian-made sports nutrition objects were used in the experiments: Whey FitPROTEIN whey protein concentrate and SPORTAMIN® BCAA 6000 branched chain amino acid carrier.

Both products have basically a similar purpose (improving body weight and tissue structure), which is believed to be associated with the activity of the FTO gene [5]. However, the level of evidence of the expected and desired effects, as well as the effect of different doses, duration reception and possible variability of the action of these SFPs on the human body has not been studied enough.

The data obtained are presented in Table 1. They show that, despite a certain similarity in the composition of the two SFPs, their effect on the average level of FTO gene expression is significantly different (daily doses of SFPs recommended by the manufacturer were used).

Table 1. Change in the average group level of FTO gene expression (normalization in relative units) in response to consumption of SPORTAMIN® BCAA 6000 and Whey FitPROTEIN.

\begin{tabular}{|c|c|c|c|c|c|c|c|}
\hline \multirow{2}{*}{ SFP under study } & \multicolumn{7}{|c|}{ Time after taking SFP, hour } \\
\cline { 2 - 8 } & $\mathbf{0}$ & $\mathbf{1}$ & $\mathbf{6}$ & $\mathbf{2 4}$ & $\mathbf{2 5}$ & $\mathbf{4 8}$ & $\mathbf{7 2}$ \\
\hline SPORTAMIN $\mathbb{B}$ & $0.20 \pm 0.02$ & 0.66 & 0.20 & 0.20 & 0.59 & 0.20 & 0.20 \\
BCAA 6000 & control & \pm 0.05 & \pm 0.05 & \pm 0.05 & \pm 0.05 & \pm 0.05 & \pm 0.05 \\
\hline Whey & $0.39 \pm 0.05$ & 0.51 & 1.55 & 0.80 & 0.71 & 0.82 & 0.75 \\
FitPROTEIN & control & \pm 0.09 & \pm 0.11 & \pm 0.09 & \pm 0.09 & \pm 0.09 & \pm 0.09 \\
\hline
\end{tabular}

It was found that consumption of BCAAs leads to an increase in the expression of the FTO gene within an hour after administration $(0.66 \pm 0.05)$ and this level decreases to normal (control) after 6 hours. Subsequently, during the entire experiment, a similar dependence is observed: a relatively fast burst of expression and the same rapid return to its usual value. 
Thus, the observed effects are short-term. It should be noted that the dynamics of BCAA action is largely consistent with the results published earlier [6].

The reaction of the FTO gene to the ingestion of Whey FitPROTEIN is of a different nature, namely, the expression reaches its maximum later, after 6 hours, and this increase is more significant than that of BCAAs, by the end of the first day it slightly decreases, but in the subsequent period of time, the increased level gene activity was maintained throughout the entire period of the experiment.

However, it should be noted that individual data on expression reveal the following phenomenon: for most of the group consuming BCAAs, the above dependence is characteristic, while in the group consuming Whey FitPROTEIN, there are two subgroups showing high and lower activity of the target gene compared to the group mean. It seems that individual differences in gene expression in subjects are explained by the presence of at least two different polymorphic variants in the FTO gene [7]. This means that a personal approach to the consumption of whey protein is required, based on a preliminary determination of the consumer's genotype (genetic testing).

The FTO gene response in all BCAAs users was approximately the same, so it can be expected that the use of BCAAs does not require a personal approach to the consumption of this SPP, and genetic testing is not desirable.

In general, summing up the results obtained in comparison with the already available data $[8,9]$, it should be stated that they supplement the known information on the effect of proteins and amino acids on the human body rather than are fundamentally new and unexpected.

In this regard, it is advisable to address the title of the article once again, and discuss the importance of using digital technologies in assessing the correctness of the experimentally obtained data on nutrigenomics and their interpretation. The development of such technologies would allow part of the scientific search to be carried out in silico.

Given the typical sequence of research, it is important to accurately select target genes for nutrients and take into account their possible polymorphism. Indeed, while the FTO gene is a target for Whey FitPROTEIN, which has been repeatedly confirmed by molecular biology methods, the situation for BCAAs is not that unambiguous. In particular, indications of the association of BCAAs with the SLC25A44 gene have appeared relatively recently [10]. Moreover, the effects of many nutrients can be realized through the functioning of a group of interacting genes. There is no doubt that digital technology like Big Data is a promising tool for solving the problem of identifying the desired gene [11].

Taking into account the possible polymorphism of target genes, one cannot fail to point out the unexpected homogeneity of the FTO gene activity in response to BCAA consumption that we discovered. It seems to us that to explain both this and similar phenomena caused by polymorphism, it would be advisable in the future to also use a methodological approach based on Big Data [12].

Another methodological difficulty of nutrigenomics is the processing of quantitative data on gene expression. On the one hand, high-throughput DNA sequencing makes it possible to obtain such data both on microarrays and on the basis of transcriptome profiling. On the other hand, their processing, which requires computer solutions for clustering, classification and association, will have to be based on digital technologies. It is here, as it seems to opinion leaders $[13,14]$, that data mining will play a leading role.

\section{Conclusion}

Thus, the algorithm for assessing the effectiveness of SFPs based on nutrigenomic studies at the achieved methodological level looks as follows.

- Study of the literature data on the relationship (association) of metabolism with the human gene(s), which is the target(s) for the action of a certain SFP. 
- Determination of the possible polymorphism of the gene associated with SFP by modern methods of molecular biology.

- Experimental study of the effect of SFP on the expression of the associated gene. This leads to obtaining own data on the dose-effect relationship and the duration of the action of the SFP on the human body to achieve the effect.

- Assessment of the degree of variability in the expression of target genes under the influence of SFPs at the individual and group levels.

- Development of recommendations for consumption (or refusal from consumption) of SFPs by individuals, including depending on their genotype in the presence of high variability of the registered effects.

As can be seen from the above, the estimated volume of labor-intensive laboratory tests is quite large and costly. Therefore, the prospect of transferring experimentation to the virtual sphere through digital technologies, in particular, digital twins, seems to be highly desirable. It will make it possible not only experimentally, but also virtually to test both existing and projected SFPs according to various criteria of a nonlinear nature and to reliably assess their effectiveness. Presumably, in the future, the Internet of Things with its smart sensors will come to the aid of digital twins. The created health sensors are already capable of measuring numerous body parameters, which are demonstrated by projects such as Philips SmartSleep headband, Polar H10, Fitbit Ionic, and this together gives a digital picture of human health and allows to reasonably judge the resulting effect of both nutrition and any other factors.

\section{References}

1. D. Samchuk, Geny i kletki, 4 (2015)

2. S. Anders, W. Huber, BioMed, 11 (2010)

3. B. Björnsson, C. Borrebaeck, N. Elander, Genome Med, 12, 4 (2020)

4. Z. Lin, M. Zamanighomi, T. Daley, S. Ma and W.H. Wong, Statist. Sci., 35 (2020)

5. D.C. Merritt, J. Jamnik, A. El-Sohemy, Genes Nutr., 13:4 (2018)

6. S. R. Jackman, O. C. Witard, A. Philp, G. A. Wallis, K. Baar and K. D. Tipton, Front. Physiol., 8: 390 (2017)

7. N. S. Guest, J. Horne, S. M. Vanderhout and A. El-Sohemy, Front. Nutr., 6:8 (2019)

8. D. D. Luis, R. Aller, O. Izaola, D. Primo, S. Urdiales, E. Romero, Lifestyle Genomics, 8 (2015)

9. F. Naclerio, E.Larumbe-Zabala, Sports Med., 46 (2016)

10. T. Yoneshiro, Q. Wang, K. Tajima, M. Matsushita, Nature, 572(7771) (2019)

11. JC. C. Wei, W. C. Chang and T. Mushiroda, Front. Pharmacol., 11:1239 (2020)

12. A. R. Sonawane, S. T. Weiss, K. Glass and A. Sharma, Front. Genet. (2019)

13. A. AlAjlan, G. Badr, ICDM 2015: Advances in Data Mining: Applications and Theoretical Aspects, 9165 (2015)

14. S. Anitha, C. P. Chandran, Bonfring Int. J. of Data Mining, 6:2 (2016) 